\title{
Task-related, low-frequency task-residual, and resting state activity in the default mode network brain regions
}

\author{
Sheng Zhang ${ }^{\text {* }}$ and Chiang-Shan R. Li ${ }^{1,2,3}$ \\ 1 Department of Psychiatry, Yale University, New Haven, CT, USA \\ 2 Department of Neurobiology, Yale University, New Haven, CT, USA \\ ${ }^{3}$ Interdepartmental Neuroscience Program, Yale University, New Haven, CT, USA
}

\section{Edited by:}

David James Sharp, Imperial College London, UK

Reviewed by:

Sven-Erik Fernaeus, Karolinska

Institutet, Sweden

David Soto, Imperial College London, UK

*Correspondence:

Sheng Zhang, Connecticut Mental Health Center S103, 34 Park Street, New Haven, CT 06519, USA.

e-mail: sheng.zhang@yale.edu
The hypothesis of a default mode network (DMN) of brain function is based on observations of task-independent decreases of brain activity during effort as participants are engaged in tasks in contrast to resting. On the other hand, studies also showed that DMN regions activate rather than deactivate in response to task-related events. Thus, does DMN "deactivate" during effort as compared to resting? We hypothesized that, with high-frequency event-related signals removed, the task-residual activities of the DMN would decrease as compared to resting. We addressed this hypothesis with two approaches. First, we examined DMN activities during resting, task residuals, and task conditions in the stop signal task using independent component analysis (ICA). Second, we compared the fractional amplitude of low-frequency fluctuation (fALFF) signals of DMN in resting, task residuals, and task data. In the results of ICA of 76 subjects, the precuneus and posterior cingulate cortex (PCC) showed increased activation during task as compared to resting and task residuals, indicating DMN responses to task events. Precuneus but not the PCC showed decreased activity during task residual as compared to resting. The latter finding was mirrored by fALFF, which is decreased in the precuneus during task residuals, as compared to resting and task. These results suggested that the low-frequency blood oxygen leveldependent signals of the precuneus may represent a useful index of effort during cognitive performance.

Keywords: posterior cingulate cortex, precuneus, default mode network, independent component analysis, spontaneous fluctuation, functional magnetic resonance imaging

\section{INTRODUCTION}

There is growing evidence from functional imaging that neural activity during a state of "resting" represents an intrinsic baseline in the default mode network (DMN), including the posterior cingulate cortex (PCC), precuneus, medial prefrontal cortex (MPFC), and inferior parietal lobule (IPL; Gusnard et al., 2001a,b; Raichle et al., 2001; Greicius et al., 2003; Fransson, 2005, 2006; Damoiseaux et al., 2006; Fox and Raichle, 2007; Buckner et al., 2008). During a resting state, these brain regions showed higher regional cerebral blood flow (CBF), local temporal synchrony, and fluctuation amplitude compared to other areas (Zou et al., 2009). The idea of a DMN was supported by two original (Shulman et al., 1997; Mazoyer et al., 2001) and subsequently confirmed by a third (Shannon, 2006) meta-analyses showing that these brain regions routinely decrease activity during task performance, as compared to resting. Thus, this DMN activity is often referred to as task-induced deactivation (TID) because the regions appear deactivated on a cognitive task. For instance, TIDs in several of the default mode regions varied parametrically as a function of task difficulty (McKiernan et al., 2003). Fransson (2006) showed that spontaneous signal fluctuations in the $\mathrm{DMN}$ persist and reorganize in response to changes in external work load.

It was hypothesized that these task-independent decreases in $\mathrm{CBF}$ are caused by interruption of ongoing processes during resting state (Shulman et al., 1997; Gusnard et al., 2001a; Mazoyer et al., 2001). On the other hand, some studies suggested that blood oxygen level-dependent (BOLD) signal changes within the DMN could result from respiration- and cardiac-induced variation (Wise et al., 2004; Birn et al., 2006; Shmuel et al., 2007; Chang et al., 2009; van Buuren et al., 2009). Several studies challenged the latter hypothesis by demonstrating a neuronal origin of DMN deactivation. For instance, a recent work employed calibrated fMRI to determine changes in the cerebral metabolic rate of oxygen $\left(\mathrm{CMRO}_{2}\right)$ and $\mathrm{CBF}$ in the DMN during a cognitive task (Lin et al., 2011). The observations that DMN regions showed a linear $\mathrm{CMRO}_{2} / \mathrm{CBF}$ relationship of neuronal activity and $\mathrm{CMRO}_{2} / \mathrm{CBF}$ ratios identical to the task-related regions confirmed the neuronal basis of DMN deactivation. The authors identified two modes of DMN deactivation each associated with more and less effort, respectively.

However, the idea of task-independent deactivation of the default brain circuit was wrought with other challenges (Morcom and Fletcher, 2007). Studies showed that behavioral tasks also elicit activity increases rather than decreases in the DMN compared to resting or passive fixation (summarized in Table 1). For instance, the PCC, anterior cingulate cortex (ACC), and precuneus respond to both pursuit and saccadic eye movement as compared to fixation in an oculomotor task (Berman et al., 1999). Distributed and 
Table 1 | Studies showing that tasks elicit activation in the DMN compared to resting or passive fixation.

\begin{tabular}{|c|c|c|}
\hline Study & Regions & Contrast \\
\hline \multicolumn{3}{|l|}{ TASK > RESTING } \\
\hline Mental navigation task (Ghaem et al., 1997) & PCC, PCu & $\begin{array}{l}\text { Mental simulation of routes vs. resting; visual imagery of } \\
\text { landmarks vs. resting }\end{array}$ \\
\hline Mental imagery task (Knauff et al., 2003) & $\mathrm{PCu}$ & Spatial vs. resting; control vs. resting \\
\hline Verbal working memory task (Osaka et al., 2003) & ACC & Task vs. resting \\
\hline Go/Nogo task (Wessa et al., 2007) & Left SMG & All go/nogo conditions vs. resting \\
\hline Memory task (Andreasen et al., 1995) & ACC & Practiced word recall vs. resting; novel word recall vs. resting \\
\hline Memory task (Andreasen et al., 1999) & ACC & Silent recall of retrieved episodic memory vs. resting \\
\hline Autobiographical memory task (Fink et al., 1996) & PCC & Autobiographical sentences trail vs. resting \\
\hline Autobiographical memory task (Markowitsch et al., 2000) & PCC, ACC, PCu & Autobiographic vs. resting; fictitious vs. resting \\
\hline $\begin{array}{l}\text { Sad and happy autobiographical memory task (Markowitsch } \\
\text { et al., 2003) }\end{array}$ & $\mathrm{ACC}, \mathrm{PCu}$ & Happy vs. resting; sad vs. resting \\
\hline Pain and attention-demanding task (Davis et al., 1997) & ACC & Pain vs. resting; attention-demanding task vs. resting \\
\hline Visuo-spatial working memory task (Garavan et al., 2000) & PCC, ACC, PCu, IPL & Task vs. resting \\
\hline Attention task (Sturm et al., 2006) & ACC, PCu, IPL & $\begin{array}{l}\text { Distributed spatial attention vs. resting; focused spatial } \\
\text { attention vs. resting }\end{array}$ \\
\hline \multicolumn{3}{|l|}{ TASK $>$ FIXATION } \\
\hline Memory and judgment task (Zysset et al., 2002) & MPFC, PCu & Episodic retrieval vs. fixation; evaluative judgment vs. fixation \\
\hline Memory task (Rothmayr et al., 2007) & PCC, PCu, IPL & Verbally or non-verbally memory delay vs. fixation \\
\hline Eye movement task (Berman et al., 1999) & $\mathrm{PCC}, \mathrm{ACC}, \mathrm{PCu}$ & $\begin{array}{l}\text { Smooth pursuit vs. fixation; visually guided saccades vs. } \\
\text { fixation }\end{array}$ \\
\hline Attention and motion task (Luks and Simpson, 2004) & ACC, ANG, SMG & Cue period vs. fixation; motion vs. fixation \\
\hline
\end{tabular}

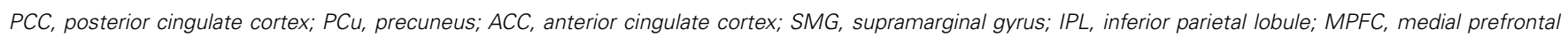
cortex; $A N G$, angular.

focused spatial attention in contrast to resting evoked activation of the ACC, precuneus, and IPL (Sturm et al., 2006).

There is growing evidence that distinguishing task-related and low-frequency BOLD signals may represent a key to disambiguating these seemingly conflicting findings. It has been suggested that baseline or spontaneous neural activity continues during a behavioral task and task-related brain activations represent a combination of the spontaneous activity and responses to stimulus inputs, behavioral outputs, and/or attention (Arfanakis et al., 2000; Fox et al., 2006a; Fair et al., 2007). In particular, Fair et al. (2007) reported that the functional connectivity of low-frequency task-residual signals (with task-elicited signals filtered, please see Methods for details) are similar to resting state. Thus, by removing task-evoked signals, one may recover spontaneous activity of the DMN during a cognitive task. In particular, one can address the possibility whether, after high-frequency signals are removed, DMN deactivate in the task residuals as compared to resting.

Indeed, our recent work showed greater activity in brain regions of the DMN - including the precuneus - during task residuals as compared to resting state in 33 adult individuals (Zhang and Li, 2010). Furthermore, we observed that the fractional amplitude of low-frequency fluctuation (fALFF) of the precuneus is greater during task residual as compared to resting. These findings support a specific role of the precuneus in mediating effort or behavioral engagement.

In the current study, we aimed to replicate and extend these earlier findings in a larger group of participants $(n=76)$. Specifically, we would show that the fALFF and the component activity (as defined by independent component analysis, ICA) of the DMN regions is decreased during task residuals as compared to resting. In contrast, we would expect greater task-evoked activation in the default brain regions as compared to both resting and task residuals.

\section{MATERIALS AND METHODS SUBJECTS AND BEHAVIORAL TASKS}

Seventy-six healthy adult subjects ( 41 men, 19-55 years of age, all right-handed) participated in four 10-min sessions of a stop signal task (SST), as described in our previous studies (Chao et al., 2009; Li et al., 2009a,b; Hendrick et al., 2010; Ide and Li, 2011 a,b; Zhang and Li, 2012a), and a 10-min resting session during fMRI. All participants were without neurological or axis I psychiatric illnesses and reported no history of head injury or use of illicit substances. In the resting state, they were instructed to relax and stay awake.

In the SST, there were two trial types: "go" and "stop," randomly intermixed. A small dot appeared on the screen to engage attention at the beginning of a go trial. After a randomized time interval (fore-period) anywhere between 1 and $5 \mathrm{~s}$, the dot turned into a circle, prompting the subjects to quickly press a button. The circle vanished at button press or after $1 \mathrm{~s}$ had elapsed, whichever came first, and the trial terminated. A premature button press prior to the appearance of the circle also terminated the trial. Three quarters of all trials were go trials. In a stop trial, an additional "X," the "stop" signal, appeared after the go signal. The subjects were told to withhold button press upon seeing the stop signal. Likewise, a trial terminated at button press or when $1 \mathrm{~s}$ had elapsed 
since the appearance of the stop signal. The stop trials constituted the remaining one quarter of the trials. There was an inter-trialinterval of $2 \mathrm{~s}$. The SSD started at $200 \mathrm{~ms}$ and varied from one stop trial to the next according to a staircase procedure, increasing and decreasing by $64 \mathrm{~ms}$ each after the successful and failed stop trial (Levitt, 1971; De Jong et al., 1990). With the staircase procedure, a "critical" SSD could be computed that represents the time delay required for the subject to succeed in withholding a response half of the time in the stop trials (Levitt, 1971). Subjects were instructed to respond to the go signal quickly while keeping in mind that a stop signal could come up in a small number of trials. Prior to the fMRI study each subject had a practice session outside the scanner. Depending on the actual stimulus timing (e.g., trials varied in foreperiod duration) and speed of response, the total number of trials varied slightly across subjects in an experiment. With the staircase procedure we anticipated that the subjects would succeed in withholding their response in approximately half of the stop trials.

\section{IMAGING PROTOCOL}

Conventional T1-weighted spin echo sagittal anatomical images were acquired for slice localization using a 3T scanner (Siemens Trio). Anatomical images of the functional slice locations were next obtained with spin echo imaging in the axial plane parallel to the AC-PC line with $\mathrm{TR}=300 \mathrm{~ms}$, $\mathrm{TE}=2.5 \mathrm{~ms}$, bandwidth $=300 \mathrm{~Hz} /$ pixel, flip angle $=60^{\circ}$, field of view $=220 \times 220 \mathrm{~mm}$, matrix $=256 \times 256$, 32 slices with slice thickness $=4 \mathrm{~mm}$ and no gap. Functional, BOLD signals were then acquired with a single-shot gradient echo echo-planar imaging (EPI) sequence. Thirty-two axial slices parallel to the AC-PC line covering the whole brain were acquired with $\mathrm{TR}=2000 \mathrm{~ms}$, $\mathrm{TE}=25 \mathrm{~ms}$, bandwidth $=2004 \mathrm{~Hz} /$ pixel, flip angle $=85^{\circ}$, field of view $=220 \mathrm{~mm} \times 220 \mathrm{~mm}$, matrix $=64 \times 64,32$ slices with slice thickness $=4 \mathrm{~mm}$ and no gap.

\section{IMAGING DATA PREPROCESSING AND GENERAL LINEAR MODELING}

Brain imaging data were preprocessed using Statistical Parametric Mapping version 8 (Wellcome Department of Imaging Neuroscience, University College London, UK). Images from the first five TRs at the beginning of each trial were discarded to enable the signal to achieve steady-state equilibrium between RF pulsing and relaxation. Images of each individual subject were first corrected for slice timing and realigned (motion corrected). A mean functional image volume was constructed for each subject for each run from the realigned image volumes. These mean images were normalized to an MNI (Montreal Neurological Institute) EPI template with affine registration followed by non-linear transformation (Friston et al., 1995a; Ashburner and Friston, 1999). The normalization parameters determined for the mean functional volume were then applied to the corresponding functional image volumes for each subject. Finally, images were smoothed with a Gaussian kernel of $8 \mathrm{~mm}$ at Full Width at Half Maximum. The data were high-pass filtered $(1 / 128 \mathrm{~Hz}$ cutoff) to remove low-frequency signal drifts.

In general linear models (GLM) events of interest were employed as regressors to explain task-related data as described in our previous work (Li et al., 2006, 2007a). Briefly, four main types of trial outcome were distinguished: go success $(\mathrm{G})$, go error $(\mathrm{F})$, stop success (SS), and stop error (SE) trial. A statistical analytical design was constructed for each individual subject, using the GLM with the onsets of go signal in each of these trial types convolved with a canonical hemodynamic response function (HRF) and with the temporal derivative of the canonical HRF and entered as regressors in the model (Friston et al., 1995b). Additional regressors with the go trial RT and stop trial SSD were also included for parametric modulation. Realignment parameters in all six dimensions were also entered in the model. The data were high-pass filtered $(1 / 128 \mathrm{~Hz}$ cutoff) to remove low-frequency signal drifts. Serial autocorrelation was corrected by a first-degree autoregressive or AR(1) model. The GLM estimated the component of variance that could be explained by each of the regressors.

Task-residual time series was obtained by removing task-related activity with the GLM. Based on previous studies that suggested a linear superposition of task activity and spontaneous BOLD fluctuations (Arfanakis et al., 2000; Fox et al., 2006a,b), it was assumed that, if task-induced variance was adequately removed, the remaining residual signal should represent the spontaneous signals (Fair et al., 2007).

\section{GROUP INDEPENDENT COMPONENT ANALYSIS}

Multivariate methods based upon ICA have been applied to examine functional connectivity between brain regions both during resting state and task performance (McKeown et al., 1998; Calhoun et al., 2001a,b, 2004, 2008; Jafri et al., 2008). Here we applied three group ICA models to the preprocessed images using the infomax algorithm, each for resting and residual, resting and task, and residual and task data (Bell and Sejnowski, 1995) as implemented in the GIFT (Group ICA of fMRI Toolbox, http://icatb.sourceforge.net/, version 2.0) software. We modified the minimum description length criteria to account for spatial correlation to determine the number of independent components (Li et al., 2007b). The three group ICA models were both estimated to have 36 components. Single subject spatial maps were reconstructed, in which the aggregate components and the results from data reduction were used to compute the individual subject components (Calhoun et al., 2001b). The DMN components were selected and the calibrated component image map of each individual subject was computed according to the percent signal change using the original fMRI data as a reference. These calibrated component image maps of all 76 subjects were examined in paired $t$ tests for resting vs. task-residual, resting vs. task, and task vs. task residual.

\section{FRACTIONAL AMPLITUDE OF LOW-FREQUENCY FLUCTUATION}

To account for power spectrum density of the low-frequency fluctuation, Zang et al. (2007) developed an index - amplitude of low-frequency fluctuation (ALFF) - in which the square root of power spectrum was integrated in a low-frequency range in order to examine the regional intensity of spontaneous BOLD fluctuations. And since the ALFF appeared to be sensitive to the physiological noise, Zou et al. (2008) proposed a fractional amplitude of low-frequency fluctuation (fALFF). We thus carried out the same fALFF analysis on resting, task-residual, and task data as we did in previous study (Zhang and Li, 2010). Briefly, filtered taskresidual and resting state time series were transformed into the frequency domain using the fast Fourier transform (FFT). Since the power is proportional to (amplitude) ${ }^{2}$ at a given frequency, the power spectrum obtained by FFT was square rooted to obtain 
amplitude. A ratio of the amplitude averaged across $0.009-0.08 \mathrm{~Hz}$ to that of the entire frequency range $(0-0.25 \mathrm{~Hz})$ was computed at each voxel to obtain the fALFF, creating an amplitude map for the whole brain, which was then normalized: normalized $\mathrm{fALFF}=(\mathrm{fALFF}-$ global mean $\mathrm{fALFF}) /$ standard deviation of global mean.

\section{RESULTS}

\section{GROUP INDEPENDENT COMPONENT ANALYSIS}

We ran group ICA 20 times using ICASSO (Himberg et al., 2004) and used cluster quality index $\left(I_{\mathrm{q}}\right)$ of the default network to assess the repeatability of ICA components. Using paired $t$ tests, we compared components of the DMN including the precuneus, PCC, and the MPFC. The results showed greater activation in the precuneus during resting as compared to task residuals $(p<0.05$, corrected for family wise error or FWE of multiple comparisons; Figure 1C; Table 2) but not when compared to task. The PCC showed less activation during resting as compared to task $(p<0.05$, FWE corrected; Figure 1F; Table 2$)$. Both the precuneus and PCC showed greater activity during task as compared to task residual ( $p<0.05$, FWE corrected; Figure 1I; Table 2$)$.

We extracted the effect size ( $z$ scores) of activity of the precuneus and PCC for each of the four SST sessions: for resting vs. residual model (Figure 2A), for resting vs. task model (Figure 2B),

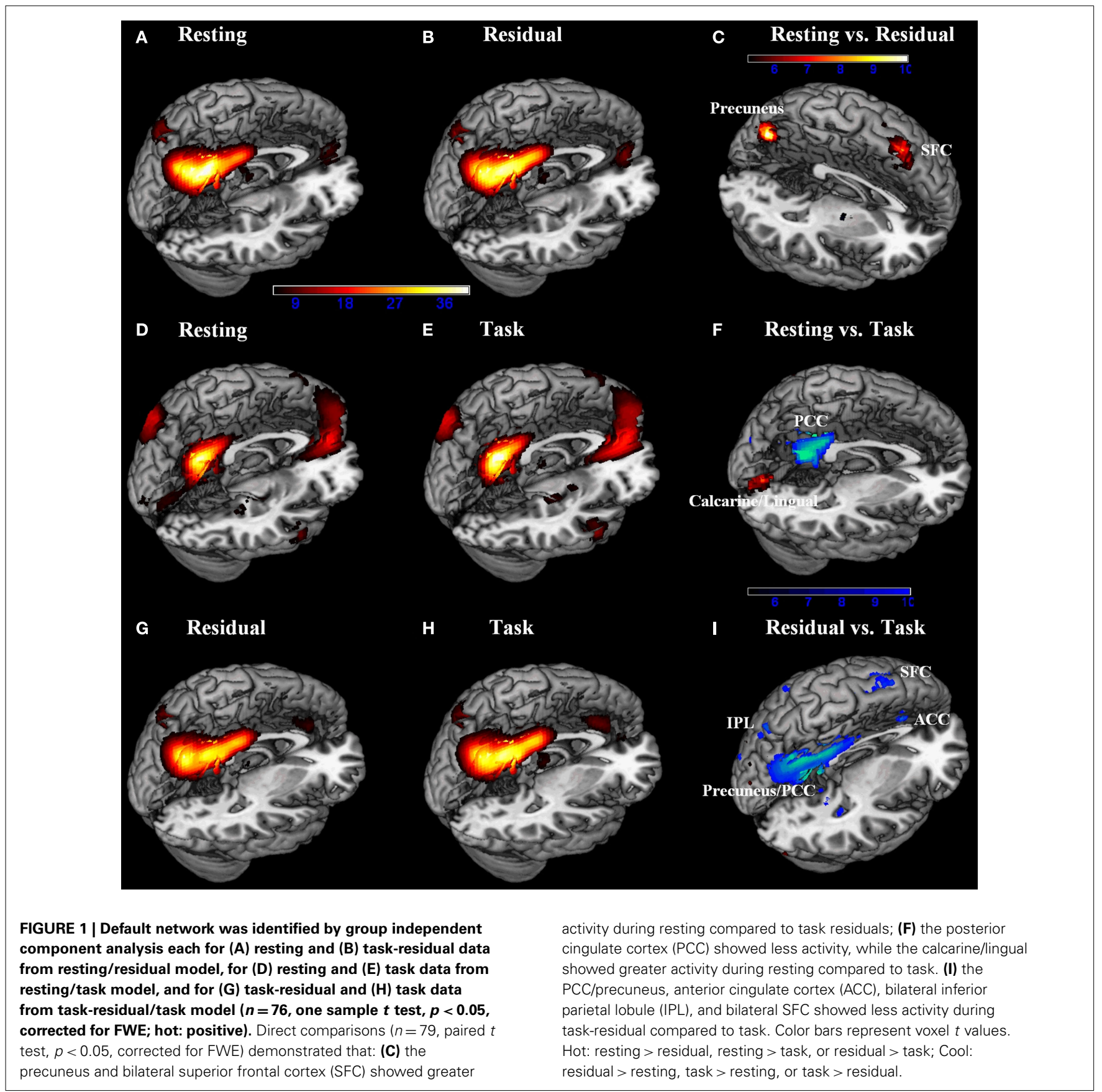


Table 2 | Regional brain activations of default network during resting compared to residual, during resting compared to task, and during residual compared to task.

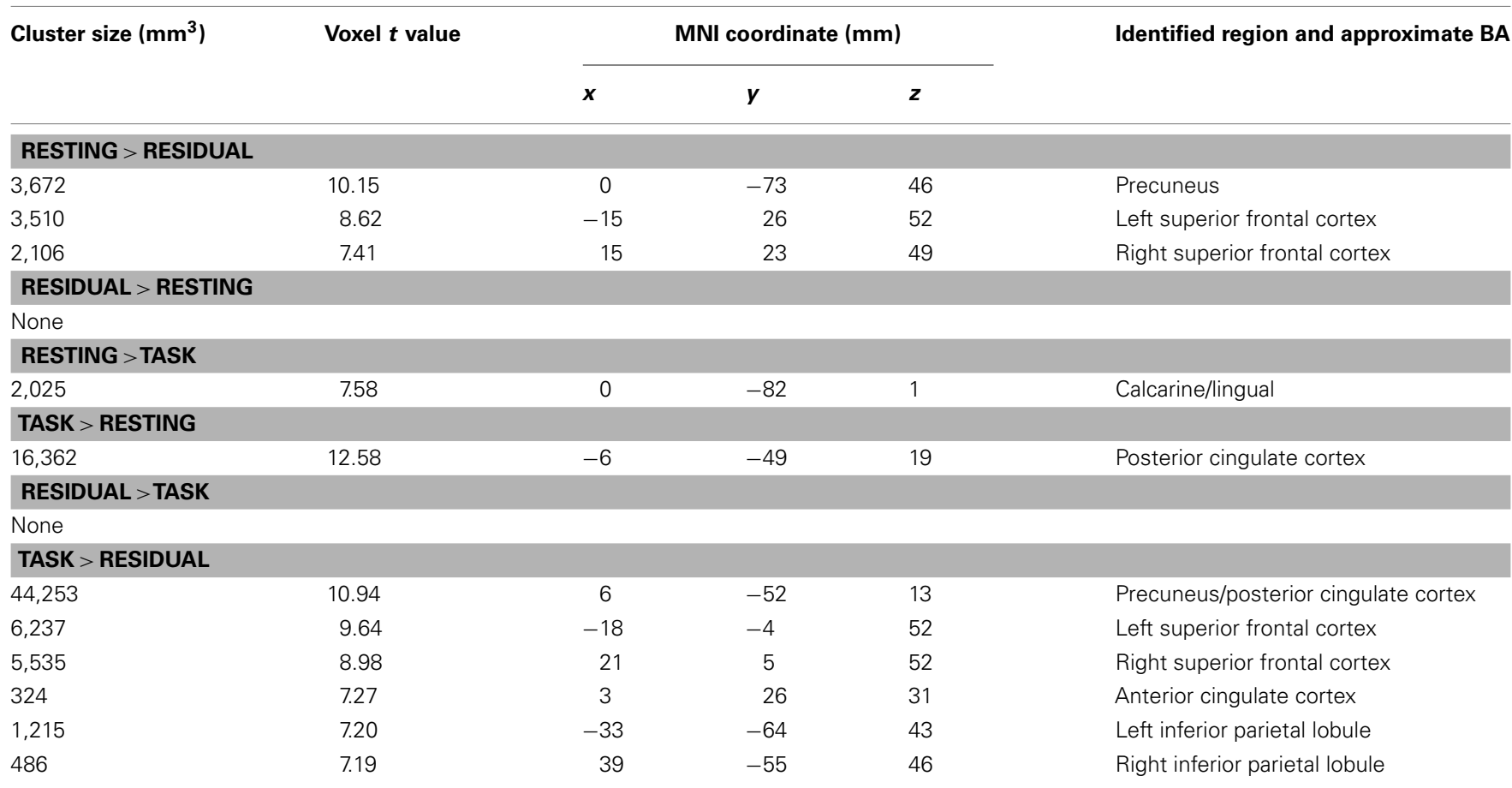

and for residual vs. task model (Figure 2C). Task residuals showed significant lower activity in the precuneus for all sessions compared to resting state (all $p<0.0001$, paired $t$ test). In contrast, task data showed significant higher activity in the PCC for all sessions compared to resting state (all $p<0.0001$, paired $t$ test). Across four sessions, task-residual precuneus activity increased across sessions (planned pair-wise comparisons: session $1<2$, $p=0.002$; session $2<3, p=0.3$; session $3<4, p=0.05$ ). In contrast, PCC activity during task decreased across the four sessions (planned pair-wise comparisons: session $1>2, p=0.002$; session $2>3, p=0.01$; session $3>4, p=0.06$ ).

In linear regressions, we correlated the component weight of the precuneus with SST performance measures. The results showed a negative correlation with the median go trial RT $(p=0.0035$, $r=-0.33)$ and a positive correlation with the go trial success $(\mathrm{RT}<1 \mathrm{~s})$ rate $(p=0.0007, r=0.38)$. These findings suggest that greater engagement is associated with more cautious responses in the SST (see Discussions).

\section{FRACTIONAL AMPLITUDE OF LOW-FREOUENCY FLUCTUATION}

In region of interest analyses, the precuneus showed greater fALFF during resting compared to task residuals $(p<0.05$, two-tailed paired $t$ test), but less fALFF during resting compared to task Figure 3). In contrast, the PCC showed less fALFF during resting compared to either task residual or task (all $p<0.001$ ).

\section{DISCUSSION}

\section{TASK-RESIDUAL LOW-FREQUENCY ACTIVITY OF THE PRECUNEUS AND} MENTAL EFFORT

The current findings from a large data set confirmed our hypothesis that task-residual low-frequency activity in the precuneus represents a neural surrogate of task engagement (Zhang and Li, 2010). The more subjects were engaged in the task, the less lowfrequency activity was observed in the precuneus. The current results support the activity of task residuals of the DMN as a neural analog of effort. Indexed by the activity effect size from ICA and the fALFF, the dorsal precuneus decreased in activity during task residuals as compared to resting. The precuneus increased activity during the task, as compared to resting. Only in task residuals, where task-related signals are removed, can we observe decreased activity in the precuneus, as compared to resting. This result is consistent with many previous findings (Gusnard et al., 2001a; McKiernan et al., 2003, 2006; Fransson, 2006). For instance, Fransson (2006) showed that spontaneous intrinsic activity in the DMN is attenuated during performance of a working memory task compared to resting. More recently, a study of traumatic brain injury (TBI) patients showed that sustained attention impairments were associated with an increase in DMN activity, particularly within the precuneus and PCC (Bonnelle et al., 2011). Another work showed that deactivation of the DMN regions including the precuneus is inversely associated with the directional coherence of the moving dots, associating DMN deactivation with effort in visual motion discrimination (Singh and Fawcett, 2008).

Evidence is accumulating to support the idea that the lowfrequency spontaneous signals are not random noise but functionally organized activity that occurs both during resting and during exposure to stimuli and cognitive challenges (Raichle et al., 2001; Greicius et al., 2003; Fox et al., 2006b; Hampson et al., 2006; Fair et al., 2007; Fox and Raichle, 2007; Raichle and Snyder, 2007). Our findings confirmed this view in that activities in the PCC, precuneus, MPFC, and IPC are organized and persists in the task-residual and task data as a network (Raichle et al., 2001; 
A Resting vs. Residual
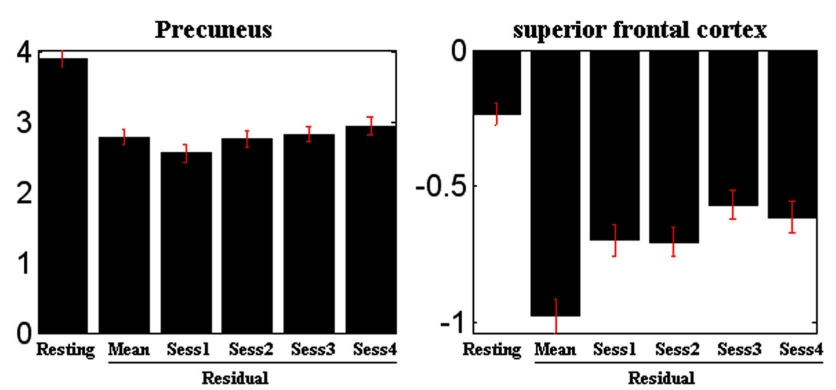

C Residual vs. Task

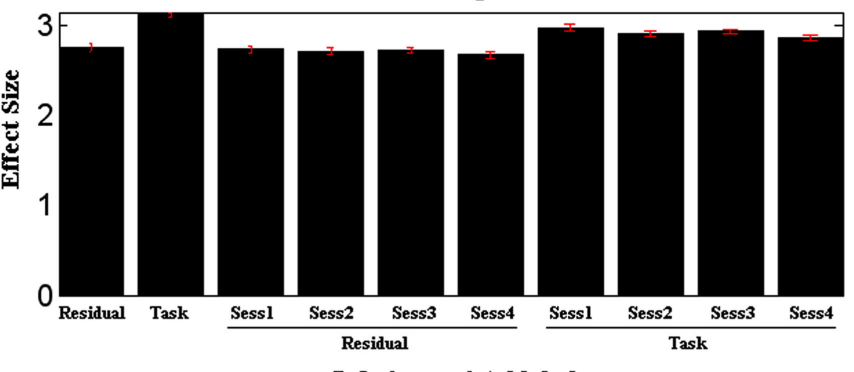

Inferior parietal lobule

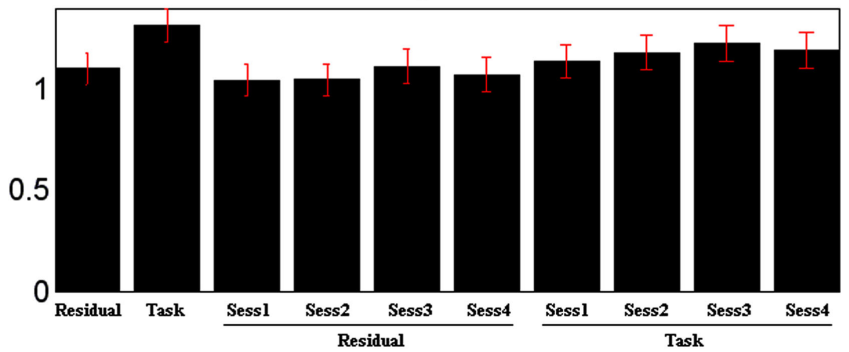

FIGURE 2 | Effect size of ROls' activity (Table 2) plotted separately for each session for (A) resting vs. residual model, (B) resting vs. task model, and (C) residual vs. task model. Task residual activity of the
B Resting vs. Task
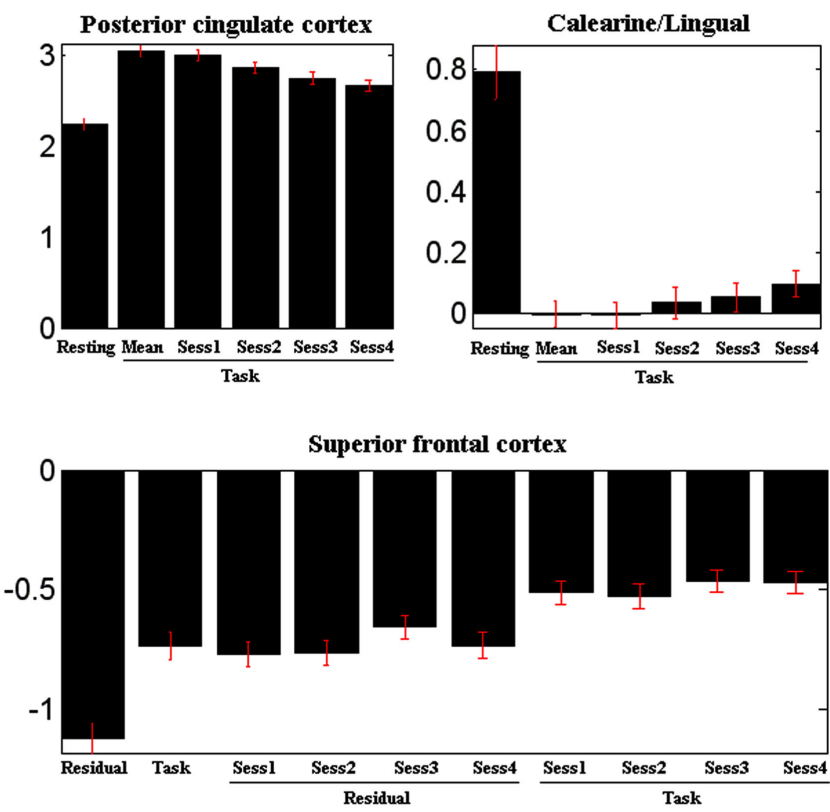

Anterior cingulate cortex

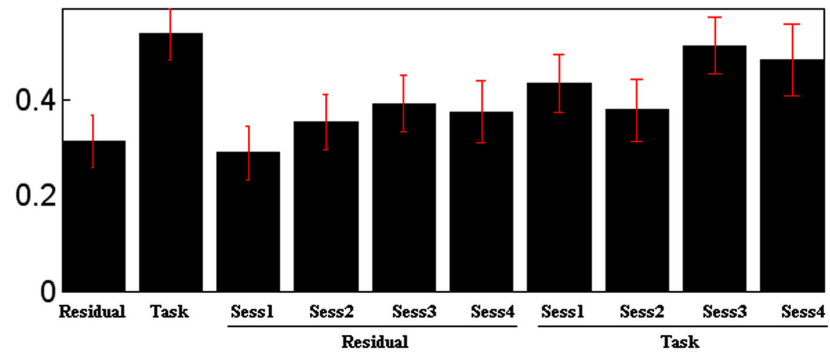

precuneus increases and task activity of the PCC decreases across sessions. Vertical bars represent standard error of the mean. See text for statistics.

increased across the four sequential sessions, suggesting that participants gradually adapt engage less mental effort to the task. In contrast, task-related activations in the PCC showed the opposite pattern of responses (see below). These results suggest an interaction of effort- and task-related activations of the DMN.

\section{DMN RESPONSES TO BOTH TASK EVENTS AND EFFORT}

The default brain regions could activate rather than deactivate in response to task-related events. We observed greater activation in the PCC during task as compared to resting state, consistent with many earlier findings obtained with different behavioral paradigms (Fink et al., 1996; Ghaem et al., 1997; Berman et al., 1999; Garavan et al., 2000; Markowitsch et al., 2000). A study showed that working memory performance was positively associated with functional connectivity between the PCC and MPFC not only during the task but also at rest (Hampson et al., 2006). The authors suggested that these regions may serve multiple roles rather than only disengage during cognitive tasks, a view that have been affirmed by other studies (Gilbert et al., 2007; Leech et al., 2011, 2012; Pearson et al., 2011). 

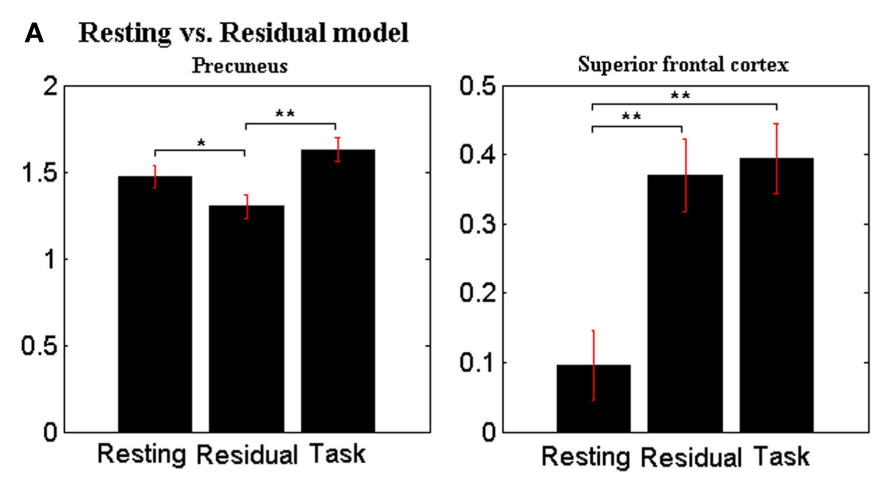

B Resting vs. Task model
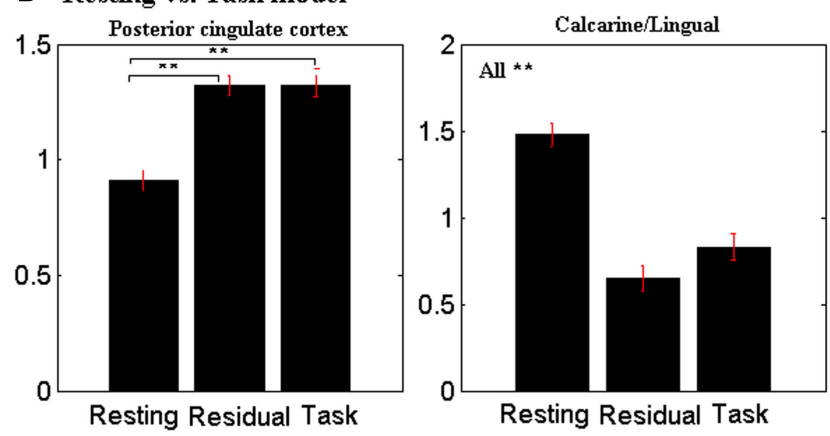

C Residual vs. Task model
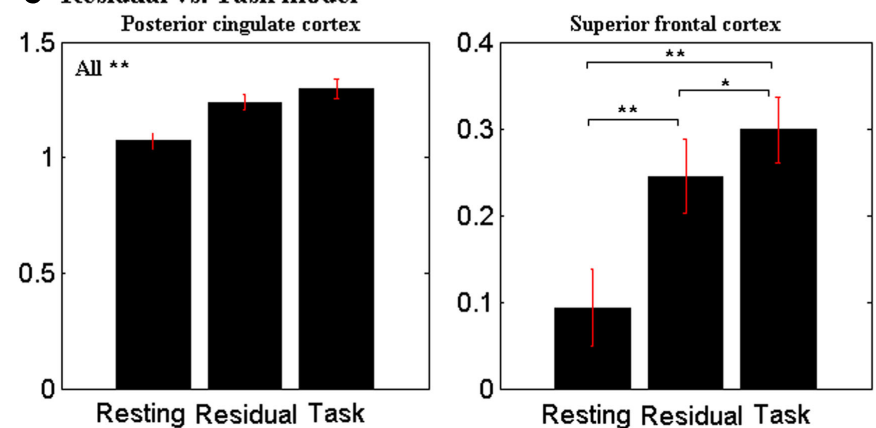
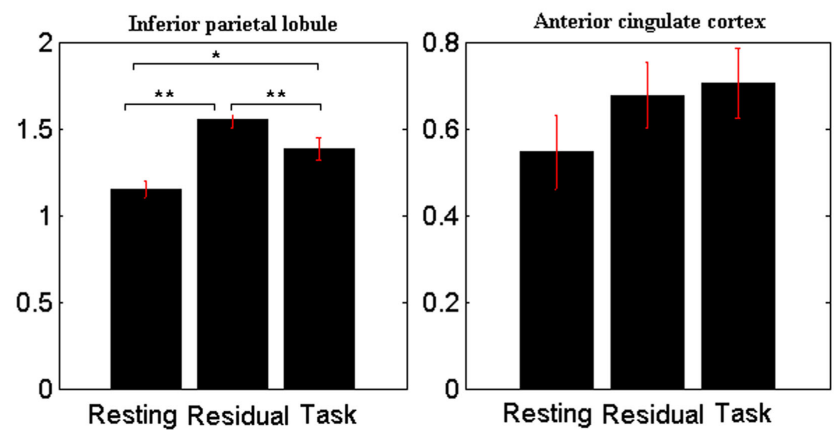

FIGURE 3 | Fractional amplitude of low-frequency fluctuation (fALFF) of observed ROls (Table 2) during resting, task-residual, and task for (A) resting vs. residual model, (B) resting vs. task model, and (C) residual vs. task model. Vertical bars represent standard error of the mean. ${ }^{*} p<0.001,{ }^{*} p<0.05$.

Notably, the observed PCC activation during task as compared to resting in our study is within the dorsal PCC. These results are consistent with two recent studies showing a dissociated pattern of activations between dorsal and ventral PCC in an attentionally demanding task (Leech et al., 2011, 2012). The dorsal PCC showed functional connectivity with both the DMN and the task-related network, while the ventral PCC showed connectivity only with the DMN. Furthermore, the PCC was suggested as an important connectivity hub by both functional and structural studies (Hagmann et al., 2008; Tomasi and Volkow, 2011). Tomasi and Volkow (2011) proposed that the PCC performs multimodal information transfer and integration, which is essential for processing spontaneous thoughts and internal awareness. Hagmann et al. (2008) investigated structural connectivity by diffusion tensor imaging, and identified a structural core comprising posterior medial and parietal cortical regions that are densely interconnected and topologically central. Taken together, these results suggest that the dorsal PCC may play an active role in the regulation of cognitive functioning besides default network activity.

Perhaps because of the mixture of responses to task-related events and effort, many previous studies reported seemingly inconsistent findings on DMN activation during cognitive performance. For instance, the activity of the default regions, such as the PCC, decreased along with the increased memory load (McKiernan et al., 2003). However, the authors observed an opposite pattern for the precuneus. A possible explanation might be that the precuneus was activated by the task while behavioral engagement caused deactivation at the same time. Such taskevoked precuneus activity compared to resting or fixation condition have been widely reported (Garavan et al., 2000; Markowitsch et al., 2000; Zysset et al., 2002; Rothmayr et al., 2007). This contrasting pattern of responses of the PCC and precuneus to the working memory task appears to be the opposite of our current findings. Thus, whether a DMN region activates more to task-related events or deactivates more to behavioral engagement may be task-specific. This issue warrants investigation in the future.

\section{CONCLUSION AND LIMITATION}

Thus, task engagement will attenuate spontaneous activity in the DMN while task-related events may increase its activity. The observed signal changes are due to a simple superposition of the spontaneous and task-evoked activity (Fox and Raichle, 2007). Consistent with this hypothesis, our findings suggest that taskresidual plays an important role in task engagement. Identification of task residual signals helps elucidate changes in spontaneous activity of the DMN, which may represent a neural signature of effort during cognitive performance.

An important issue remains to be resolved. The precuneus that we identified as the neural surrogate of effort is within the dorsal part of the medial posterior parietal cortex. Our recent study of connectivity mapping showed that the ventral but not the dorsal precuneus is within the default network (Zhang and Li, 2012b, see also Buckner et al., 2008). In contrast, dorsal precuneus is connected to brain regions in control of attention and mental 
imagery. A recent study supported this functional distinction by showing decreased regional CBF in the posterior cingulate gyrus, medial frontal cortex, and ventral but not dorsal precuneus, in participants performing a spatial working memory task, as compared to rest (Pfefferbaum et al., 2011). Thus, although both the dorsal and ventral precuneus appear to be involved in behavioral engagement to an external task, their specific roles remained to be specified.

\section{REFERENCES}

Andreasen, N. C., O'Leary, D. S., Cizadlo, T., Arndt, S., Rezai, K., Watkins, G. L., Ponto, L. L., and Hichwa, R. D. (1995). II. PET studies of memory: novel versus practiced free recall of word lists. Neuroimage 2, 296-305.

Andreasen, N. C., O'Leary, D. S., Paradiso, S., Cizadlo, T., Arndt, S., Watkins, G. L., Ponto, L. L., and Hichwa, R. D. (1999). The cerebellum plays a role in conscious episodic memory retrieval. Hum. Brain Mapp. 8, 226-234.

Arfanakis, K., Cordes, D., Haughton, V. M., Moritz, C. H., Quigley, M. A., and Meyerand, M. E. (2000). Combining independent component analysis and correlation analysis to probe interregional connectivity in fMRI task activation datasets. Magn. Reson. Imaging 18, 921-930.

Ashburner, J., and Friston, K. J. (1999). Nonlinear spatial normalization using basis functions. Hum. Brain Mapp. 7, 254-266.

Bell, A. J., and Sejnowski, T. J. (1995). An information maximisation approach to blind separation and blind deconvolution. Neural Comput. 7, 1129-1159.

Berman, R. A., Colby, C. L., Genovese, C. R., Voyvodic, J. T., Luna, B., Thulborn, K. R., and Sweeney, J. A. (1999). Cortical networks subserving pursuit and saccadic eye movements in humans: an fMRI study. Hum. Brain Mapp. 8, 209-225.

Birn, R. M., Diamond, J. B., Smith, M. A., and Bandettini, P. A. (2006). Separating respiratoryvariation-related fluctuations from neuronal-activity-related fluctuations in fMRI. Neuroimage 31, 1536-1548.

Bonnelle, V., Leech, R., Kinnunen, K. M., Ham, T. E., Beckmann, C. F., De Boissezon, X., Greenwood, R. J., and Sharp, D. J. (2011). Default mode network connectivity predicts sustained attention deficits after traumatic brain injury. J. Neurosci. 31, 13442-13451.
Buckner, R. L., Andrews-Hanna, J. R., and Schacter, D. L. (2008). The brain's default network: anatomy, function, and relevance to disease. Ann. N. Y. Acad. Sci. 1124, 1-38.

Calhoun, V. D., Adali, T., McGinty, V. B., Pekar, J. J., Watson, T. D., and Pearlson, G. D. (2001a). fMRI activation in a visual-perception task: network of areas detected using the general linear model and independent components analysis. Neuroimage 14, 1080-1088.

Calhoun, V. D., Adali, T., Pearlson, G. D., and Pekar, J. J. (2001b). A method for making group inferences from functional MRI data using independent component analysis. Hum. Brain Mapp. 14, 140-151.

Calhoun, V. D., Adali, T., and Pekar, J. J. (2004). A method for comparing group fMRI data using independent component analysis: application to visual, motor and visuomotor tasks. Magn. Reson. Imaging 22, 1181-1191.

Calhoun, V. D., Kiehl, K. A., and Pearlson, G. D. (2008). Modulation of temporally coherent brain networks estimated using ICA at rest and during cognitive tasks. Hum. Brain Mapp. 29, 828-838.

Chang, C., Cunningham, J. P., and Glover, G. H. (2009). Influence of heart rate on the BOLD signal: the cardiac response function. Neuroimage 44, 857-869.

Chao, H. H., Luo, X., Chang, J. L., and Li, C. S. (2009). Activation of the pre-supplementary motor area but not inferior prefrontal cortex in association with short stop signal reaction time - an intra-subject analysis. BMC Neurosci. 10, 75 . doi:10.1186/1471-2202-10-75

Damoiseaux, J. S., Rombouts, S. A., Barkhof, F., Scheltens, P., Stam, C. J., Smith, S. M., and Beckmann, C. F. (2006). Consistent resting-state networks across healthy subjects. Proc. Natl. Acad. Sci. U.S.A. 103, 13848-13853.

Davis, K. D., Taylor, S. J., Crawley, A. P., Wood, M. L., and Mikulis, D. J. (1997). Functional MRI of pain-and

\section{ACKNOWLEDGMENTS}

This study was supported by NIH grants R01DA023248, R21AA018004, K02DA026990. We thank Sarah Bednarski, Emily Erdman, Sien Hu, and Olivia Farr in subject recruitment and assessment as well as running of the imaging studies. The NIH had no further role in study design; in the collection, analysis, and interpretation of data; in the writing of the report; or in the decision to submit the paper for publication.

attention-related activations in the human cingulate cortex. J. Neurophysiol. 77, 3370-3380.

De Jong, R., Coles, M. G., Logan, G. D., and Gratton, G. (1990). In search of the point of no return: the control of response processes. J. Exp. Psychol. Hum. Percept. Perform. 16, 164-182. Fair, D. A., Schlaggar, B. L., Cohen, A. L., Miezin, F. M., Dosenbach, N. U., Wenger, K. K., Fox, M. D., Snyder, A. Z., Raichle, M. E., and Petersen, S. E. (2007). A method for using blocked and event-related fMRI data to study "resting state" functional connectivity. Neuroimage 35, 396-405.

Fink, G. R., Markowitsch, H. J., Reinkemeier, M., Bruckbauer, T., Kessler, J. and Heiss, W. D. (1996). Cerebral representation of one's own past: neural networks involved in autobiographical memory. J. Neurosci. 16, 4275-4282.

Fox, M. D., and Raichle, M. E. (2007). Spontaneous fluctuations in brain activity observed with functional magnetic resonance imaging. Nat. Rev. Neurosci. 8, 700-711.

Fox, M. D., Snyder, A. Z., Zacks, J. M., and Raichle, M. E. (2006a). Coherent spontaneous activity accounts for trial-to-trial variability in human evoked brain responses. Nat. Neurosci. 9, 23-25.

Fox, M. D., Corbetta, M., Snyder, A. Z., Vincent, J. L., and Raichle, M. E. (2006b). Spontaneous neuronal activity distinguishes human dorsal and ventral attention systems. Proc. Natl. Acad. Sci. U.S.A. 103, 10046-10051.

Fransson, P. (2005). Spontaneous lowfrequency BOLD signal fluctuations: an $\mathrm{fMRI}$ investigation of the restingstate default mode of brain function hypothesis. Hum. Brain Mapp. 26 15-29.

Fransson, P. (2006). How default is the default mode of brain function? Further evidence from intrinsic BOLD signal fluctuations. Neuropsychologia 44, 2836-2845.

Friston, K., Ashburner, J., Frith C., Polone, J., Heather, J., and Frackowiak, R. (1995a). Spatial registration and normalization of images. Hum. Brain Mapp. 2, 165-189.

Friston, K., Holmes, A. P., Worsley, K. J., Poline, J. B., Frith, C., and Frackowiak, R. (1995b). Statistical parametric maps in functional imaging: a general linear approach. Hum. Brain Mapp. 2, 189-210.

Garavan, H., Kelley, D., Rosen, A., Rao, S. M., and Stein, E. A. (2000). Practicerelated functional activation changes in a working memory task. Microsc. Res. Tech. 51, 54-63.

Ghaem, O., Mellet, E., Crivello, F., Tzourio, N., Mazoyer, B., Berthoz, A., and Denis, M. (1997). Mental navigation along memorized routes activates the hippocampus, precuneus, and insula. Neuroreport 8, 739-744.

Gilbert, S. J., Dumontheil, I., Simons, J. S., Frith, C. D., and Burgess, P. W. (2007). Comment on "Wandering minds: the default network and stimulus-independent thought". Science 317, 43; author reply 43.

Greicius, M. D., Krasnow, B., Reiss, A. L., and Menon, V. (2003). Functional connectivity in the resting brain: a network analysis of the default mode hypothesis. Proc. Natl. Acad. Sci. U.S.A. 100, 253-258.

Gusnard, D. A., Raichle, M. E., and Raichle, M. E. (2001a). Searching for a baseline: functional imaging and the resting human brain. Nat. Rev. Neurosci. 2, 685-694.

Gusnard, D. A., Akbudak, E., Shulman, G. L., and Raichle, M. E. (2001b) Medial prefrontal cortex and selfreferential mental activity: relation to a default mode of brain function. Proc. Natl. Acad. Sci. U.S.A. 98, 4259-4264.

Hagmann, P., Cammoun, L., Gigandet, X., Meuli, R., Honey, C. J., Wedeen, V. J., and Sporns, O. (2008). Mapping the structural core of human cerebral cortex. PLoS Biol. 6, e159. doi:10.1371/journal.pbio.0060159

Hahn, B., Ross, T. J., and Stein, E. A. (2007). Cingulate activation increases dynamically with response speed under stimulus unpredictability. Cereb. Cortex 17, 1664-1671. 
Hampson, M., Driesen, N. R., Skudlarski, P., Gore, J. C., and Constable, R. T. (2006). Brain connectivity related to working memory performance. J. Neurosci. 26, 13338-13343.

Hendrick, O. M., Ide, J. S., Luo, X., and $\mathrm{Li}, \mathrm{C}$. S. (2010). Dissociable processes of cognitive control during error and non-error conflicts: a study of the stop signal task. PLoS ONE 5, e13155. doi:10.1371/journal.pone.0013155

Himberg, J., Hyvarinen, A., and Esposito, F. (2004). Validating the independent components of neuroimaging time series via clustering and visualization. Neuroimage 22, 1214-1222.

Ide, J. S., and Li, C. S. (2011a). A cerebellar thalamic cortical circuit for error-related cognitive control. Neuroimage 54, 455-464.

Ide, J. S., and Li, C. S. (2011b). Error-related functional connectivity of the habenula in humans. Front. Hum. Neurosci. 5:25. doi:10.3389/fnhum.2011.00025

Jafri, M. J., Pearlson, G. D., Stevens, M., and Calhoun, V. D. (2008). A method for functional network connectivity among spatially independent resting-state components in schizophrenia. Neuroimage 39, 1666-1681.

Knauff, M., Fangmeier, T., Ruff, C. C., and Johnson-Laird, P. N. (2003). Reasoning, models, and images: behavioral measures and cortical activity. J. Cogn. Neurosci. 15, 559-573.

Leech, R., Braga, R., and Sharp, D. J. (2012). Echoes of the brain within the posterior cingulate cortex. J. Neurosci. 32, 215-222.

Leech, R., Kamourieh, S., Beckmann, C. F., and Sharp, D. J. (2011). Fractionating the default mode network: distinct contributions of the ventral and dorsal posterior cingulate cortex to cognitive control. J. Neurosci. 31, 3217-3224.

Levitt, H. (1971). Transformed updown methods in psychoacoustics. J. Acoust. Soc. Am. 49(Suppl. 2), 467.

Li, C. S., Huang, C., Constable, R. T., and Sinha, R., (2006). Imaging response inhibition in a stopsignal task: neural correlates independent of signal monitoring and post-response processing. J. $\mathrm{Neu}$ rosci. 26, 186-192.

Li, C. S., Chao, H. H., and Lee, T. W. (2009a). Neural correlates of speeded as compared with delayed responses in a stop signal task: an indirect analog of risk taking and association with an anxiety trait. Cereb. Cortex 19, 839-848.
Li, C. S., Zhang, S., Duann, J. R., Yan, P., Sinha, R., and Mazure, C. M. (2009b). Gender differences in cognitive control: an extended investigation of the stop signal task. Brain Imaging Behav. 3, 262-276.

Li, C. S., Yan, P., Bergquist, K. L., and Sinha, R., (2007a). Greater activation of the "default" brain regions predicts stop signal errors. Neuroimage 38, 640-648.

Li, Y. O., Adali, T., and Calhoun, V. D. (2007b). Estimating the number of independent components for functional magnetic resonance imaging data. Hum. Brain Mapp. 28, 1251-1266.

Lin, P., Hasson, U., Jovicich, J., and Robinson, S. (2011). A neuronal basis for task-negative responses in the human brain. Cereb. Cortex 21 , 821-830.

Luks, T. L., and Simpson, G. V. (2004). Preparatory deployment of attention to motion activates higher-order motion-processing brain regions. Neuroimage 22, 1515-1522.

Markowitsch, H. J., Thiel, A., Reinkemeier, M., Kessler, J., Koyuncu, A., and Heiss, W. D. (2000). Right amygdalar and temporofrontal activation during autobiographic, but not during fictitious memory retrieval. Behav. Neurol. 12, 181-190.

Markowitsch, H. J., Vandekerckhove, M. M., Lanfermann, H., and Russ, M. O. (2003). Engagement of lateral and medial prefrontal areas in the ecphory of sad and happy autobiographical memories. Cortex 39, 643-665.

Mazoyer, B., Zago, L., Mellet, E., Bricogne, S., Etard, O., Houde, O., Crivello, F., Joliot, M., Petit, L., and Tzourio-Mazoyer, N. (2001). Cortical networks for working memory and executive functions sustain the conscious resting state in man. Brain Res. Bull. 54, 287-298.

McKeown, M. J., Makeig, S., Brown, G. G., Jung, T. P., Kindermann, S. S., Bell, A. J., and Sejnowski, T. J. (1998). Analysis of fMRI data by blind separation into independent spatial components. Hum. Brain Mapp. 6, 160-188.

McKiernan, K. A., D’Angelo, B. R., Kaufman, J. N., and Binder, J. R. (2006). Interrupting the "stream of consciousness": an fMRI investigation. Neuroimage 29, 1185-1191.

McKiernan, K. A., Kaufman, J. N., Kucera-Thompson, J., and Binder, J. R. (2003). A parametric manipulation of factors affecting taskinduced deactivation in functional neuroimaging. J. Cogn. Neurosci. 15 394-408.

Morcom, A. M., and Fletcher, P. C. (2007). Does the brain have a baseline? Why we should be resisting a rest. Neuroimage 37, 1073-1082.

Osaka, M., Osaka, N., Kondo, H., Morishita, M., Fukuyama, H., Aso, T. and Shibasaki, H. (2003). The neural basis of individual differences in working memory capacity: an fMRI study. Neuroimage 18, 789-797.

Pearson, J. M., Heilbronner, S. R. Barack, D. L., Hayden, B. Y., and Platt, M. L. (2011). Posterior cingulate cortex: adapting behavior to a changing world. Trends Cogn. Sci. (Regul. Ed.) 15, 143-151.

Pfefferbaum, A., Chanraud, S., Pitel, A. L., Muller-Oehring, E., Shankaranarayanan, A., Alsop, D. C., Rohlfing, T., and Sullivan, E. V. (2011). Cerebral blood flow in posterior cortical nodes of the default mode network decreases with task engagement but remains higher than in most brain regions. Cereb. Cortex 21, 233-244.

Raichle, M. E., Macleod, A. M., Snyder, A. Z., Powers, W. J., Gusnard, D. A., and Shulman, G. L. (2001). A default mode of brain function. Proc. Natl. Acad. Sci. U.S.A. 98, 676-682.

Raichle, M. E., and Snyder, A. Z. (2007). A default mode of brain function: a brief history of an evolving idea. Neuroimage 37, 1083-1090; discussion 1097-1089.

Rothmayr, C., Baumann, O., Endestad, T., Rutschmann, R. M., Magnussen, S., and Greenlee, M. W. (2007). Dissociation of neural correlates of verbal and non-verbal visual working memory with different delays. Behav. Brain Funct. 3, 56.

Shannon, B. J. (2006). Functional Anatomic Studies of Memory Retrieval and the Default Mode. PhD thesis, Washington University in St. Louis, 184.

Shmuel, A., Yacoub, E., Chaimow, D., Logothetis, N. K., and Ugurbil, K. (2007). Spatio-temporal pointspread function of fMRI signal in human gray matter at 7 Tesla. $\mathrm{Neu}$ roimage 35, 539-552.

Shulman, G. L., Fiez, J. A., Corbetta, M., Buckner, R. L., Miezin, F. M., Raichle, M. E., and Petersen, S. E. (1997). Common blood flow changes across visual tasks: II. Decreases in cerebral cortex. J. Cogn. Neurosci. 9, 648-663.

Singh, K. D., and Fawcett, I. P. (2008). Transient and linearly graded deactivation of the human default-mode network by a visual detection task. Neuroimage 41, 100-112.
Sturm, W., Schmenk, B., Fimm, B., Specht, K., Weis, S., Thron, A., and Willmes, K. (2006). Spatial attention: more than intrinsic alerting? Exp. Brain Res. 171, 16-25.

Tomasi, D., and Volkow, N. D. (2011). Association between functional connectivity hubs and brain networks. Cereb. Cortex 21, 2003-2013.

van Buuren, M., Gladwin, T. E., Zandbelt, B. B., van den Heuvel, M., Ramsey, N. F., Kahn, R. S., and Vink, M. (2009). Cardiorespiratory effects on default-mode network activity as measured with fMRI. Hum. Brain Mapp. 30, 3031-3042.

Wessa, M., Houenou, J., PaillereMartinot, M. L., Berthoz, S., Artiges, E., Leboyer, M., and Martinot, J. L. (2007). Fronto-striatal overactivation in euthymic bipolar patients during an emotional go/nogo task. Am. J. Psychiatry 164, 638-646.

Wise, R. G., Ide, K., Poulin, M. J., and Tracey, I. (2004). Resting fluctuations in arterial carbon dioxide induce significant low frequency variations in BOLD signal. Neuroimage 21, 1652-1664.

Zang, Y. F., He, Y., Zhu, C. Z., Cao, Q. J., Sui, M. Q., Liang, M., Tian, L. X., Jiang, T. Z., and Wang, Y. F. (2007). Altered baseline brain activity in children with $\mathrm{ADHD}$ revealed by resting-state functional MRI. Brain Dev. 29, 83-91.

Zhang, S., and Li, C. S. (2010). A neural measure of behavioral engagement: task-residual low-frequency blood oxygenation level-dependent activity in the precuneus. Neuroimage 49 , 1911-1918.

Zhang, S., and Li, C. S. (2012a). Functional networks for cognitive control in a stop signal task: independent component analysis. Hum. Brain Mapp. 33, 89-104.

Zhang, S., and Li, C. S. (2012b). Functional connectivity mapping of the human precuneus by resting state fMRI. Neuroimage 59, 3548-3562.

Zou, Q. H., Wu, C. W., Stein, E. A., Zang, Y. F., and Yang, Y. (2009). Static and dynamic characteristics of cerebral blood flow during the resting state. Neuroimage 48 , 515-524.

Zou, Q. H., Zhu, C. Z., Yang, Y., Zuo, X. N., Long, X. Y., Cao, Q. J., Wang, Y. F., and Zang, Y. F. (2008). An improved approach to detection of amplitude of low-frequency fluctuation (ALFF) for resting-state fMRI: fractional ALFF. J. Neurosci. Methods 172, 137-141 
Zysset, S., Huber, O., Ferstl, E., and von Cramon, D. Y. (2002). The anterior frontomedian cortex and evaluative judgment: an fMRI study. Neuroimage 15, 983-991.

Conflict of Interest Statement: The authors declare that the research was conducted in the absence of any commercial or financial relationships that could be construed as a potential conflict of interest.

Received: 24 March 2012; paper pending published: 22 April 2012; accepted: 13 May 2012; published online: 30 May 2012.
Citation: Zhang $S$ and $\mathrm{Li}$ C-SR (2012) Task-related, low-frequency taskresidual, and resting state activity in the default mode network brain regions. Front. Psychology 3:172. doi: 10.3389/fpsyg.2012.00172

This article was submitted to Frontiers in Cognition, a specialty of Frontiers in Psychology.
Copyright (c) 2012 Zhang and Li. This is an open-access article distributed under the terms of the Creative Commons Attribution Non Commercial License, which permits noncommercial use, distribution, and reproduction in other forums, provided the original authors and source are credited. 